DRAFT VERSION JANUARY 7, 2019

Preprint typeset using LATEX style AASTeX6 v. 1.0

\title{
UNDERSTANDING FORMATION OF YOUNG, DISTRIBUTED LOW-MASS STARS AND CLUSTERS IN THE
} W4 CLOUD COMPLEX

\author{
Neelam Panwar ${ }^{1,3}$, Manash R. Samal ${ }^{2}$, A. K. Pandey ${ }^{1}$, H. P. Singh ${ }^{3}$, Saurabh Sharma ${ }^{1}$ \\ ${ }^{1}$ Aryabhatta Research Institute of Observational Sciences (ARIES), Nainital - 263001, India \\ ${ }^{2}$ Physical Research Laboratory, Navrangpura, Ahmedabad - 380009, India \\ ${ }^{3}$ Department of Physics \& Astrophysics, University of Delhi, Delhi-110007, India
}

\begin{abstract}
It is well known that most of the stars form in rich clusters. However, recent Spitzer observations have shown that a significant number of stars also form in distributed mode, origin of which is not well understood. In this work, we aim to investigate clustered and distributed mode of star formation in the W4 complex. To do so, we identified and characterized the young stellar population associated with the region using homogeneous infra-red data-sets obtained from 2MASS, GLIMPSE, MIPS and WISE surveys. We make stellar surface density and minimum spanning tree maps to identify young clusters, and use Spitzer images to identify irradiated structures, such as elephant trunk-like structures (ETLSs) and pillars in the region. The surface density distribution of the young stellar objects (YSOs) reveals three new clusterings and $\sim 50 \%$ distributed protostars in the HII region. The clusters are of low-mass nature but significantly younger than the central cluster IC 1805. We identified $\sim 38$ ETLSs in the region, a majority of which consist of one or a few stars at their tips. We find these stars are low-mass $\left(<2 \mathrm{M}_{\odot}\right)$ YSOs, located at the outskirts $(>17 \mathrm{pc})$ of the cluster IC 1805 and are part of scattered distributed population. We argued that the star formation in the ETLSs of W4 is going on possibly due to triggering effect of expanding W4 bubble. Although high-resolution photometric and spectroscopic data would be required to confirm the scenario, nonetheless, we discuss the implications of this scenario for our understanding of distributed low-mass star formation in cloud complexes as opposed to other mechanisms such as turbulent fragmentation and dynamical ejection.
\end{abstract}

Keywords: stars : formation - stars : pre-main-sequence - ISM : globules Ây HII regions - open cluster: initial mass function; star formation.

\section{INTRODUCTION}

Observations of many star forming regions (SFRs) have shown that cluster or group is the dominant mode of star formation. Stellar clustering results from the fractal properties of the molecular clouds under the effects of turbulence (Elmegreen \& Efremov 1997; Elmegreen et al. 2014). Hence, star clusters can be used to investigate star formation from small scales (in small dense clouds) to the larger scales (in giant molecular clouds). At the same time, mid-infrared surveys such as Spitzer have shown that significant number of stars form in a more distributed mode. For example, Evans et al. (2009) studied several nearby molecular clouds and found that in each cloud a population of isolated young stellar objects (YSOs) is distributed throughout the cloud (see also Koenig et al. 2008). The origin of this distributed population is unclear so far. It is also not clear under what circumstances distributed mode of star formation would be more prevalent.

In this work we aim to understand the formation of clusters and distributed mode of star formation by investigating young stellar content of the $\mathrm{W} 4$ complex. The W4 complex (see Fig. 1), located at a distance of $\sim 2$ kpc in the Perseus arm of the Galaxy, is a part of the $\mathrm{W} 3 / \mathrm{W} 4 / \mathrm{W} 5$ cloud complex. It is ionized by the massive members of the cluster IC 1805 (Massey et al. 1995). The cluster IC 1805 is located at the central part of the W4 HII region and embedded in a very low-extinction cloud of mean $E(B-V) \sim 0.8$ mag (see Panwar et al. 2017, and references therein). It is a young cluster with an age of $\sim 2.5 \mathrm{Myr}$ and total mass of $\sim 2700 \mathrm{M}_{\odot}$ (Panwar et al. 2017; Sung et al. 2017). It harbors dozens of massive stars (O- or early B-type), of which the three most massive are $\mathrm{BD}+60502(\mathrm{O} 4.5 \mathrm{III}), \mathrm{BD}+60501(\mathrm{O} 7 \mathrm{~V})$ and HD 15570 (O4I) (Massey et al. 1995; Lefloch \& Lazareff 1995), hence more energetic than the Orion Nebula (e.g., see discussion in Panwar et al. 2018). These massive members of the cluster IC 1805 have created a central cavity of $\sim 40 \mathrm{pc}$ through mechanical and radiation output. In fact Spitzer and Herschel images of the His region 
show that it has a bipolar bubble morphology (Panwar et al. 2017), similar to that found by Deharveng et al. (2015); Samal et al. (2018). The ionizing sources have a considerable effect on the structure of the molecular material as W4 bubble contains many bright-rimmed clouds (BRCs), namely, BRC 5, BRC 6, BRC 7, BRC 8, BRC 9 and a cometary globule $\mathrm{CG} 7 \mathrm{~S}$, which are thought to arise from the impact of UV photons of nearby massive stars on pre-existing dense molecular material (Sugitani et al. 1991; Lefloch \& Lazareff 1995; Morgan et al. 2004; Miao et al. 2009; Bisbas et al. 2011). Most of these BRCs contain Class 0/I sources at their heads which may be the products of triggering due to radiation pressure from the massive members of the cluster IC 1805. The age distributions of the YSOs as a function of the distance from the most massive stars of the IC 1805) in BRCs 5 and 7 manifest age gradients (Panwar et al. 2014) supporting the model of triggered sequential star formation in these clouds.

This paper is organized as follows. We describe the datasets used in the present work in Sec. 2. Sec. 3 describes identification of candidate YSOs in the HiI region. We discuss the incidence of clusterings and isolated distribution of YSOs in the region and also their possible origin in Sec. 4. In Sec. 5, we provide conclusions of our study of the clustered and distributed mode of star formation in the complex and our main results.

\section{DATA USED}

The W4 bubble was observed in $24 \mu \mathrm{m}$ using the Multiband Imaging Photometer for Spitzer (MIPS) in two epochs. However, a part of the bubble (see upper-right corner of Fig. 1) is missing in these observations. We downloaded the MIPS post basic calibrated data images from the Spitzer archive which were created at the image scale of $2^{\prime \prime} .45$ per pixel. The MOPEX-APEX pipeline was used to detect the point sources and to perform the point response function (PRF) fitting photometry. All the sources were visually examined and any ambiguous sources were removed. In addition, we also included point sources manually which were not automatically detected by APEX by supplying their co-ordinates to the Apex user-list module of MOPEX software to perform the PRF fitting and to extract the fluxes. The zero-point value of 7.14 Jy (adopted from MIPS Data Handbook) is used to convert the flux densities to magnitudes.

We also used the archival infrared array camera (IRAC) data from GLIMPSE-360 (P.I. - B. A. Whitney). The GLIMPSE-360 observations were taken during the Spitzer warm mission and hence were available only at $3.6 \mu \mathrm{m}$ and $4.5 \mu \mathrm{m}$. To ensure the good quality data, we considered sources with photometric uncertainties $\leq 0.2$ mag in each band.

The Wide-field Infrared Survey Explorer (WISE) has

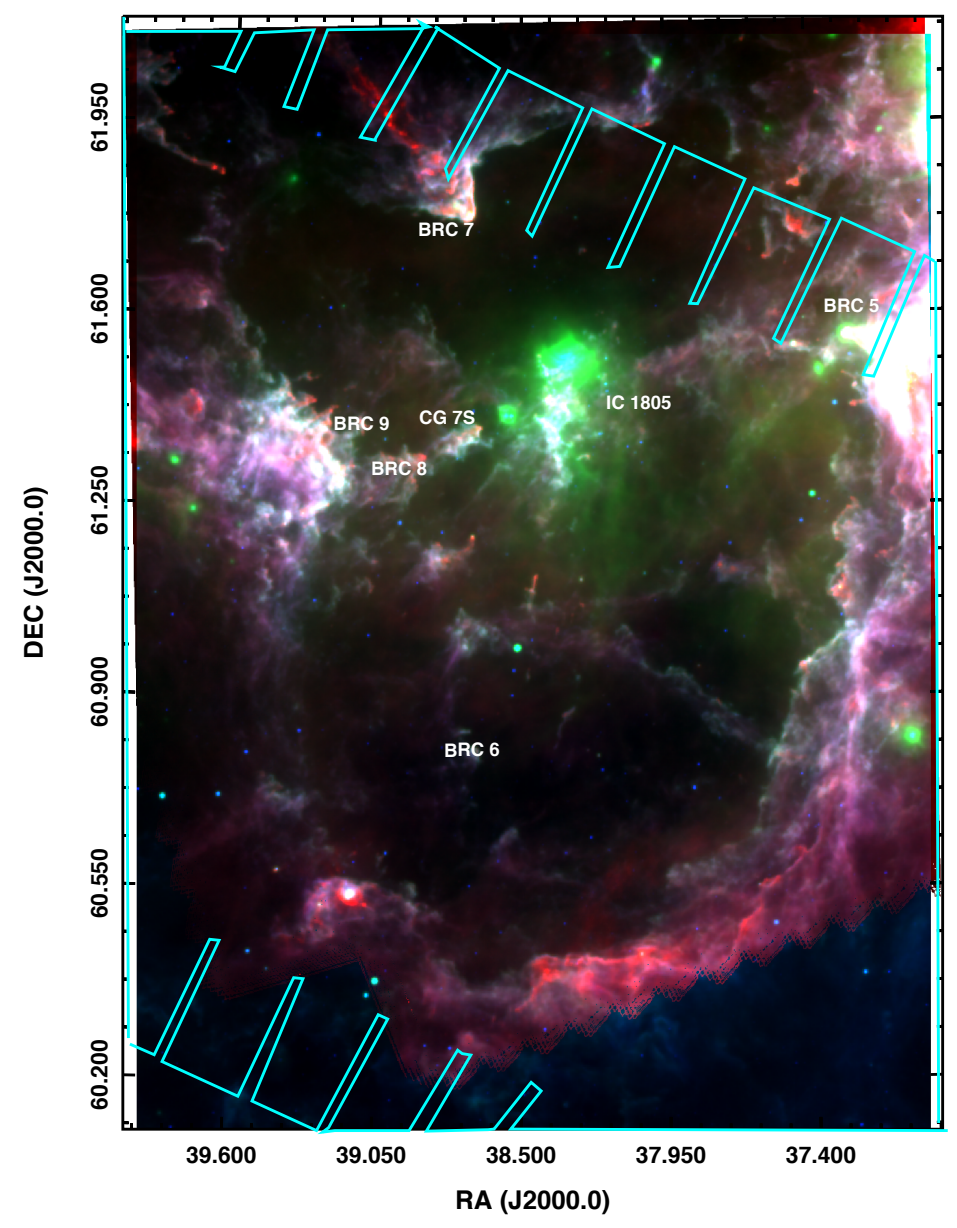

Figure 1. Color-composite image (red: Herschel $500 \mu \mathrm{m}$, green: WISE $22 \mu \mathrm{m}$ and blue: WISE $12 \mu \mathrm{m}$ ) of W4 Hı region. The locations of central ionizing cluster IC 1805 alongwith BRCs and cometary globule are also marked. Cyan polygon represents the area covered by MIPS 24 $\mu \mathrm{m}$ observations.

explored the sky at four wavebands $(3.4,4.6,12$, and $22 \mu \mathrm{m})$. We have used the WISE catalog from Cutri et al. (2014). We selected only sources that have contamination and confusion flags "000" in 3.4, 4.6 and 12 $\mu \mathrm{m}$ wavebands. NIR $J H K_{s}$ data for the stars in the HiI region have been obtained from the Two Micron All Sky Survey (2MASS) Point Source Catalog (PSC) (Cutri et al. 2003).

For making various color-color diagrams to identify YSOs in the region, we band-merge all of the above catalogues as follows. We matched the GLIMPSE data with 2MASS catalog using a maximum $1^{\prime \prime}$ radial tolerance (Gutermuth et al. 2008; Panwar et al. 2014). We integrated MIPS $24 \mu \mathrm{m}$ catalog using a $2^{\prime \prime} .5$ maximum radial tolerance with the GLIMPSE catalog (Megeath et al. 2012). In cases, where there was more than one source 
within the matching radius, the closest one is considered as the best match.

\section{ANALYSIS : IDENTIFICATION OF YOUNG STELLAR OBJECTS}

YSOs trace the most recent sites of star formation in a SFR as they are too young to undergo dynamical evolution and can be used to understand the star formation history and other properties of the region (Snider et al. 2009; Deharveng et al. 2012; Pandey et al. 2013a,b; Bernard et al. 2016; Yadav et al. 2016; Sharma et al. 2017). They exhibit excess emission in IR wavelengths due to their circumstellar disks. Hence, NIR to MIR photometric surveys of SFRs are useful tools to identify YSOs. YSOs occupy distinct positions in infrared color-color (CC) diagrams due to their distinct characteristics which can be used for identification of young stellar content of a complex. In this work, we are interested in tracing recent star formation activity of the complex. Therefore, we only searched for Class I and Class II YSOs of the complex. We describe several color diagnosis to identify such YSOs of the W4 complex.

\subsection{YSOs using 2MASS/IRAC colors}

The IRAC CC diagrams are often used to identify and classify the YSO populations in SFRs (Megeath et al. 2004; Allen et al. 2004). In the absence of IRAC higher wavelength observations, IRAC 3.6, $4.5 \mu \mathrm{m}$ data can be combined with 2MASS $H$ and $K_{s}$ data to identify YSOs of a complex (Gutermuth et al. 2008, 2009). Following Gutermuth et al. (2009), we used IRAC/2MASS intrinsic K-[3.6] / [3.6]-[4.5] color-color diagram to identify the YSOs and classify them. In order to implement this method, we first generated the extinction map for the region using 2MASS data following the procedure described in Panwar et al. (2014). In brief, we compute stellar extinction maps with the method proposed by Cambrésy et al. (2002). We divided the region into small bins and calculated the $(\mathrm{H}-\mathrm{K})$ colors of each bin as the median of the $(\mathrm{H}-\mathrm{K})$ colors of the 10 nearest neighbours. We then calculated $\mathrm{E}(H-K)=(H-$ $K)-(\mathrm{H}-\mathrm{K})_{0}$, where $(\mathrm{H}-\mathrm{K})_{0}$ is the median color of the stars in field region. We calculated $A_{K_{s}}$ for each bin using the relation $A_{K_{s}}=1.82 \times \mathrm{E}(\mathrm{H}-\mathrm{K})$. We then made an extinction map for the whole region. We dereddened all those stars detected in $\mathrm{K}, 3.6$ and $4.5 \mu \mathrm{m}$ using the extinction laws of Flaherty et al. (2007). We used the $(K-[3.6])_{0}$ and $([3.6]-[4.5])_{0}$ color cuts to select Class I and Class II sources as described in Gutermuth et al. (2009). To minimize contamination from the extragalactic sources, especially PAH emitting galaxies and active galactic nuclei (AGN) in our YSO sample, we selected objects having [3.6] < $14.5 \mathrm{mag}$ (Gutermuth et al. 2009). By this method we obtained 398 Class II and 23 Class I sources in the region. Fig. 2a shows the $([3.6]-[4.5])_{0}$ vs. $(K-[3.6])_{0}$ CC diagram for the candidate YSOs.

\subsection{Additional YSOs from $24 \mu \mathrm{m}$}

There may be some sources which are deeply embedded and were not detected at any of the 2MASS/IRAC bands but possess MIPS photometry. To identify the additional YSOs and to examine the YSOs identified using 2MASS/IRAC colours, we included the sources which have $24 \mu \mathrm{m}$ counterparts. We found that out of $\sim 791$ point sources with magnitude uncertainty $\leq 0.2$ mag within the region shown in Fig. 1, 550 MIPS sources have counterparts in GLIMPSE data. We considered sources which appear bright in MIPS $24 \mu \mathrm{m}$ ([24] $<7 \mathrm{mag})$ and have very red colors ([4.5]-[24] $>4.5 \mathrm{mag})$ as Class I sources (Gutermuth et al. 2009). We choose brighter sources as at fainter magnitudes the extragalactic contamination may dominate our YSO sample (e.g., Megeath et al. 2009; Gutermuth et al. 2009). Using these criteria, we found $\sim 92$ protostellar candidates, which are shown with magenta triangles in MIPS/IRAC colormagnitude diagram (CMD) in Fig. 2b. 43 candidates out of these were identified using 2MASS/GLIMPSE data. All the previously identified protostars having $24 \mu \mathrm{m}$ detections were checked and considered as Class II sources if they did not have ([4.5] - [24]) > 4 mag (Gutermuth et al. 2009). Out of previously identified 23 Class I sources, 6 were reclassified as Class II sources.

\subsection{YSOs from WISE data}

We also used the WISE 3.4, 4.6 and $12 \mu \mathrm{m}$ data for characterization of the embedded YSOs using the approach developed by Koenig et al. (2012). This selection method uses a series of color and magnitude cuts to remove the contaminants such as background galaxies and nebulosity blobs. We identified 142 Class II and 18 Class I sources in the region. Out of these, 106 YSOs are already identified using aforementioned schemes. Fig. $2 c$ shows the WISE CC diagram for the sources in the W4 Hir region. Green Circles and red squares represent Class I and Class II sources, respectively.

\subsection{YSOs Summary and Completeness}

Our final catalog includes the YSO candidates identified from Spitzer MIPS, GLIMPSE/2MASS and WISE data sets. We may point out that broad-line AGNs, unresolved knots of shock emission and faint sources contaminated by copious PAH nebulosity are likely to affect our YSO selection. Using the criteria of Gutermuth et al. (2009); Koenig et al. (2012), we have removed these contaminants from our YSO catalog. Although these criteria for a distant region $(\sim 2 \mathrm{kpc})$ may lead to an overestimation of the contamination, this would ensure the high reliability of our YSO catalog. Robitaille et al. (2008) found 

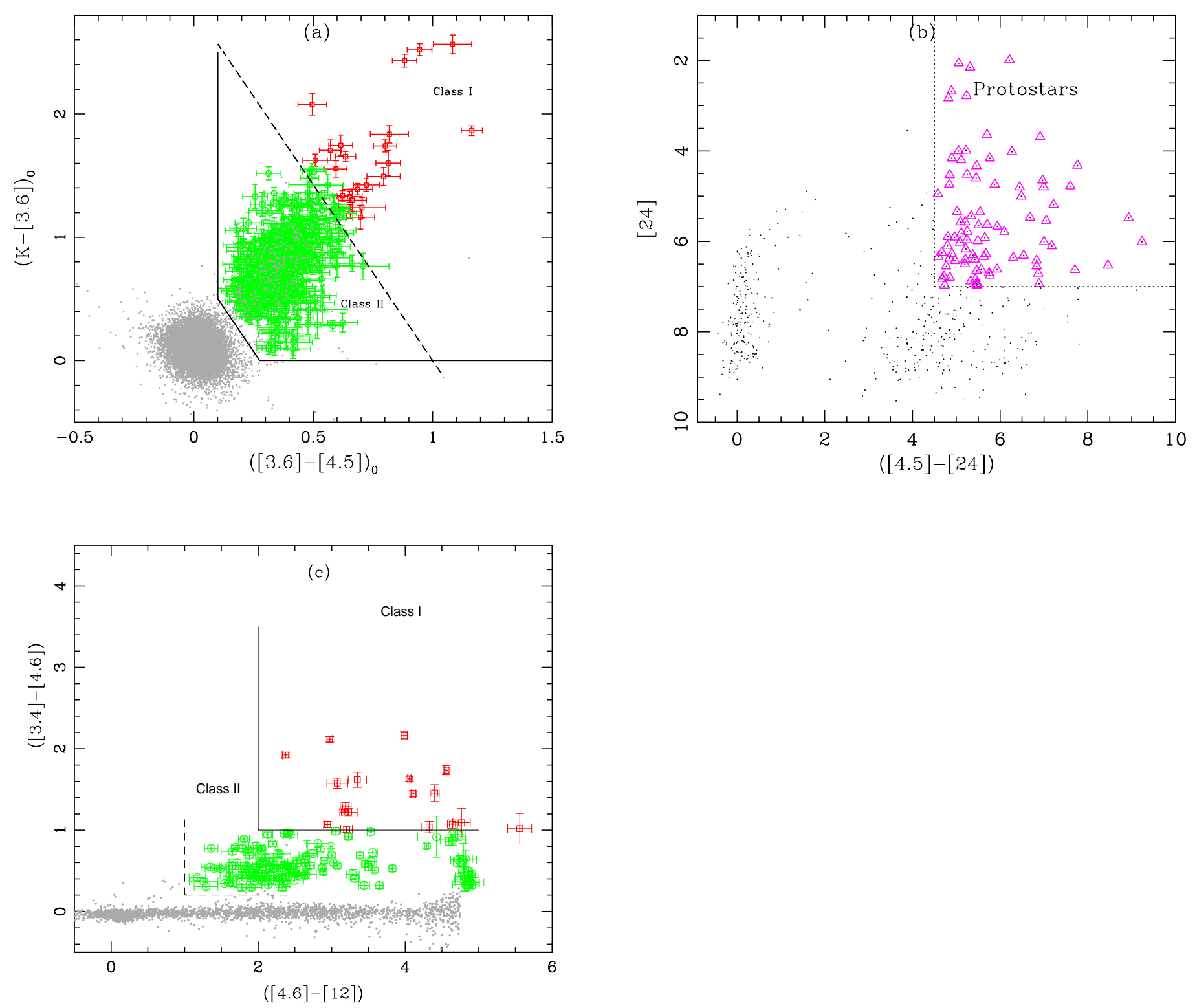

Figure 2. (a) GLIMPSE/2MASS $\left(\mathrm{K}_{s} \text { - [3.6]) }\right)_{0}$ vs. ([3.6] - [4.5]) $)_{0}$ color-color diagram, (b) [24] vs. ([4.5] - [24]) colormagnitude diagram and (c) WISE ([3.4]-[4.6])/ ([4.6]-[12.0]) color-color diagram for the sources in W4 region. Red squares represent the Class I and green circles represent Class II sources. Magenta triangles represent the protostellar sources identified based on the MIPS/GLIMPSE color-magnitude criterion.

that in their sample of sources with redder IRAC/MIPS colors, approximately 30\%-50\% may be asymptotic giant branch (AGB) stars whereas planetary nebulae and background galaxies together may be at most $2 \%-3 \%$ of the total. AGB stars show a steep spectral index at long wavelengths. Based on their SEDs, Robitaille et al. (2008) used a criteria [8.0] - [24] $<2.2$ mag to identify them. In the absence of IRAC $8.0 \mu \mathrm{m}$ data, we are unable to apply Robitaille et al. (2008) criteria to eliminate such sources. However, Robitaille et al. (2008) approxi- mated the surface density distribution of candidate AGB stars in different longitude and latitude bins by a simple function. This function suggests a negligible contribution from the AGB stars $(\sim 0.004)$ within the region covered in the present study. In total, we found $\sim 524$ candidate YSOs in the direction of the W4 complex. Out of these $\sim 100$ are Class I sources. A sample list of the YSO candidates with their magnitudes in different bands is given in Table 1 and the entire table is available in electronic form only. 
The peak of the observed luminosity function can be assumed to estimate the $90 \%$ completeness limit (Evans et al. 2003; Jose et al. 2016). In order to evaluate the completeness of the MIPS, IRAC/2MASS and WISE data, we plot the histograms of the sources detected in each band. The completeness limits are $8.0 \mathrm{mag}, 13.5$ mag and $13.0 \mathrm{mag}$ in $24 \mu \mathrm{m}, 3.6 \mu \mathrm{m}$ and $4.5 \mu \mathrm{m}$, respectively. The completeness limits of WISE data are estimated as 12.5, 12.5, 9.5 and 7.0 mag in the 3.4, 4.6, 12 and $22 \mu \mathrm{m}$ wavebands, respectively. In our analysis, we have used only first three WISE wavelengths. Therefore, the completeness of the WISE sources is limited by the bright nebulosity and relatively low sensitivity at 12 $\mu \mathrm{m}$. In this work, majority of the YSOs have been identified using IRAC/2MASS CC diagram (see Fig. 2a). We found $90 \%$ of the $3.6 \mu \mathrm{m}$ and $4.5 \mu \mathrm{m}$ sources have $\mathrm{K}_{s}$-band counterparts. Therefore, majority of the IRAC sources have been detected in $\mathrm{K}_{s}$-band. Thus our YSO completeness limit is primarily limited by the completeness of $\mathrm{K}_{s}$ band, which is $K_{s} \sim 14.5 \mathrm{mag}$. We note that the local completeness limits can be affected by the variable reddening and crowding of the sources across the region. Using the evolutionary models of Siess et al. (2000) for an age of 2 Myr (lifetime of Class II phase of YSOs) and a distance of $2 \mathrm{kpc}$, the mass completeness corresponds to $\sim 0.5 \mathrm{M}_{\odot}$.

\section{RESULTS \& DISCUSSION}

As W4 is located at a low Galactic latitude $\left(1=134^{\circ} .7\right.$, $\left.\mathrm{b}=0^{\circ} .9\right)$, the contamination due to line of sight stars, extra-galactic sources and reddened evolved stars can still be significant at shorter wavelengths although we tried to minimize it using various color or magnitude cutoffs. Therefore, we adopted different approaches to study the clustered and distributed young stellar population in the complex. Since cluster appears as an enhanced concentration of point sources in the stellar density map, we used YSO surface density map of the complex to identify young clusters Contaminating sources in a SFR can be eliminated either using spectroscopic observations or using deep wide-field optical and X-ray observations as one can evaluate the properties of candidate YSOs by comparing their locations in the HR diagram with the theoretical pre-main sequence (PMS) isochrones (for details see Panwar et al. 2017, 2018). In the absence of these observations, we primarily use bright MIPS $24 \mu \mathrm{m}$ flux to identify distributed isolated Class I population of the complex. This is done mainly to reduce the contamination from the background reddened sources and from the faint evolved sources in our likely sample of distributed mode population. As YSOs emit relatively strong $24 \mu \mathrm{m}$ emission during their early evolution phases and also extinction effects are less at $24 \mu \mathrm{m}$, the MIPS data are particularly important for classification and separation of sources from the reddened field stars. We emphasize that with the aforementioned photometric approach it is impossible to eliminate line-of-sight PMS sources (if any) those are not part of W4 complex.

\subsection{Creation of YSO Surface Density Map : Incidence of Clusterings}

We used the nearest neighbor method to generate the YSO surface density map (Gutermuth et al. 2005). In brief, we divided the region into uniform grids of $1^{\prime}$. We measured the radial distance $\mathrm{r}_{n}(\mathrm{i}, \mathrm{j})$ of the $\mathrm{n}^{\text {th }}$ nearest neighbour from each position in a uniform grid. From this radial distance a surface density is directly computed as $\sigma_{n}(\mathrm{i}, \mathrm{j})=(\mathrm{n}-1) /\left(\pi \mathrm{r}_{n}{ }^{2}(\mathrm{i}, \mathrm{j})\right.$ (Casertano \& Hut 1985). We varied the value of $n$ and after a series of experiments decided the value of $\mathrm{n}=6$, which is a good compromise between the resolution and the signal-to-noise ratio of the surface density map. The contours for the surface density calculated by the above method, plotted above $4 \sigma$ level, are shown in Fig. 3. The surface density distribution reveals five prominent density peaks in the region. One of the density peaks appears at the center of the Hir region, i.e., on the cluster IC 1805 (marked as ' 1 '). The second peak is near BRC 5/ AFGL 333, which is studied by Jose et al. (2016). The positions of other three peaks do not match with the clusters mentioned in the literature and thus are likely new cluster candidates. Below we describe details of these new clusters.

\subsubsection{Density Peak near to BRC 7}

One of the density peaks (marked as ' 3 ' in Fig. 3) is at $\alpha_{2000}=38^{\circ} .621, \delta_{2000}=+61^{\circ} .787$. It is near the apex of BRC 7. To study the stellar extent of this clustering, we plotted its radial density profile (RDP) using our YSO sample. We divided the cluster into a number of concentric circular regions. To calculate the projected stellar density in each concentric annulus, we divided the number of stars within each annular region by the respective area. The densities thus obtained are plotted as a function of radius in Fig. 4a. The error bars are derived assuming that the number of stars in each annulus follows the Poisson statistics.

We model the RDP with a function similar to the Elson, Fall \& Freeman (EFF) profile (Elson et al. 1987), often used to characterize the radial profiles of young clusters in the galaxies. The fitted function (dashed curve in Fig. 4a) is of the form:

$$
\rho(r) \propto \rho_{0}\left[1+\left(\frac{r}{a}\right)^{\beta}\right]^{-\gamma / \beta},
$$

where $\beta=2.0$ (Elson et al. 1987). $\rho_{0}$, a and $\gamma$ are the central surface density, measure of the core radius and the power-law slope at large radii, respectively. Similarly, we also fit the observed RDP with the empirical King profile (King 1962) of the form: 
Table 1. YSOs from MIPS, IRAC/2MASS and WISE data. The entire table is available in electronic form.

\begin{tabular}{|c|c|c|c|c|c|c|c|c|c|c|c|}
\hline Id & $\begin{array}{c}\text { RA } \\
(\mathrm{J} 2000)\end{array}$ & $\begin{array}{c}\text { DEC } \\
(\mathrm{J} 2000)\end{array}$ & $\mathrm{J} \pm \mathrm{eJ}$ & $\mathrm{H} \pm \mathrm{eH}$ & $K_{s} \pm \mathrm{e} K_{s}$ & $\begin{array}{l}{[3.6] \pm} \\
\mathrm{e}[3.6]\end{array}$ & $\begin{array}{l}{[4.5] \pm} \\
\mathrm{e}[4.5]\end{array}$ & $\begin{array}{c}{[24] \pm} \\
\mathrm{e}[24]\end{array}$ & $\begin{array}{l}{[3.4] \pm} \\
\mathrm{e}[3.4]\end{array}$ & $\begin{array}{l}{[4.6] \pm} \\
\mathrm{e}[4.6]\end{array}$ & $\begin{array}{c}{[12] \pm} \\
\mathrm{e}[12]\end{array}$ \\
\hline 1 & 38.42181 & 60.39177 & - & $15.79 \pm 0.17$ & $14.43 \pm 0.08$ & $13.14 \pm 0.05$ & $12.40 \pm 0.04$ & $6.94 \pm 0.05$ & $13.23 \pm 0.03$ & $12.25 \pm 0.03$ & $9.19 \pm 0.05$ \\
\hline 2 & 38.83216 & 60.43402 & $16.00 \pm 0.09$ & $14.86 \pm 0.08$ & $13.94 \pm 0.06$ & $12.28 \pm 0.04$ & $11.65 \pm 0.03$ & $7.37 \pm 0.02$ & $12.45 \pm 0.03$ & $11.68 \pm 0.02$ & $8.83 \pm 0.04$ \\
\hline 3 & 39.25507 & 60.51685 & - & $15.59 \pm 0.15$ & $12.96 \pm 0.03$ & $10.20 \pm 0.07$ & $9.05 \pm 0.04$ & $4.00 \pm 0.01$ & $10.88 \pm 0.03$ & $8.95 \pm 0.02$ & $6.58 \pm 0.04$ \\
\hline
\end{tabular}
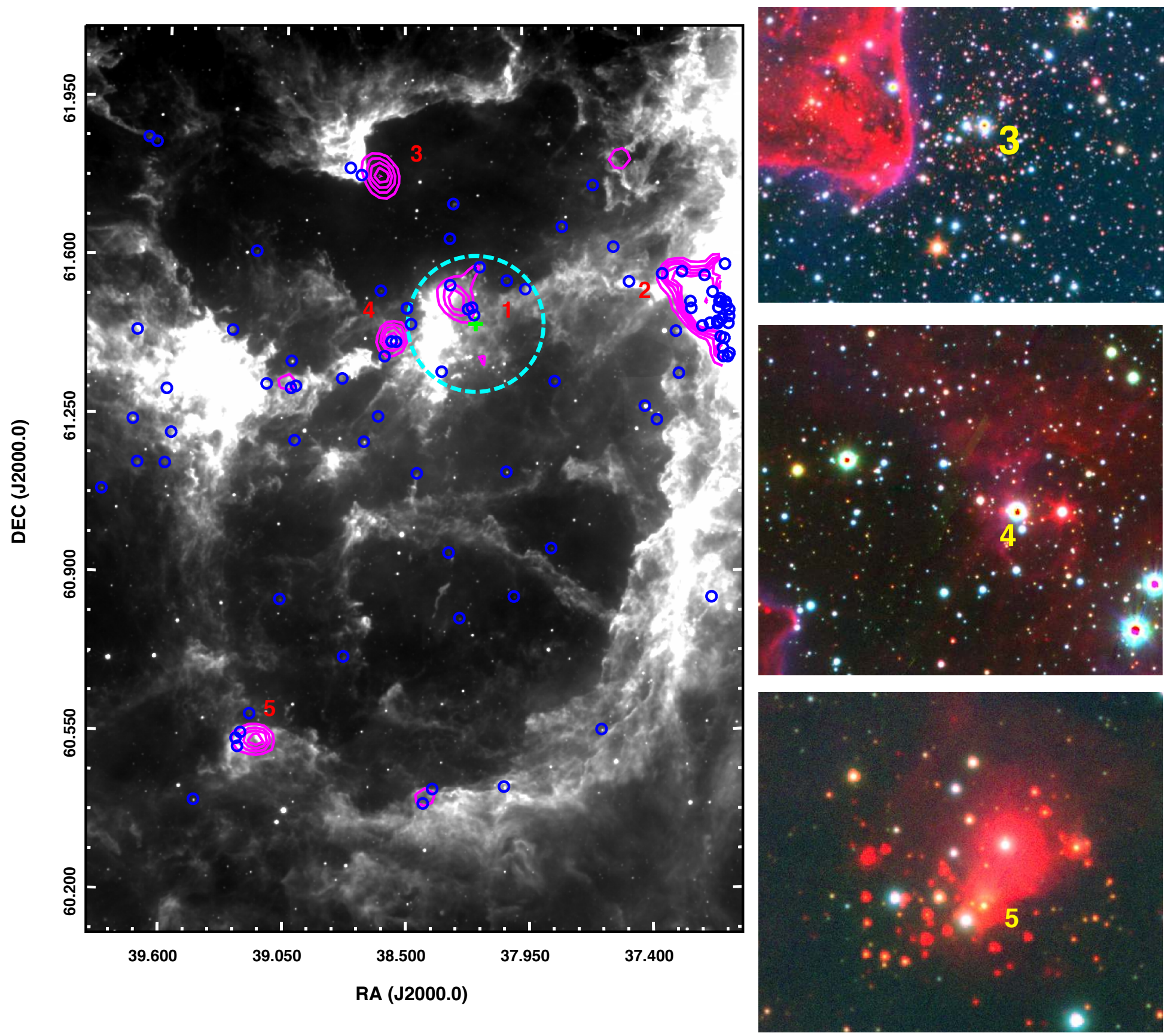

Figure 3. Left panel: Surface density contours of the identified YSOs overlaid on $22 \mu \mathrm{m}$ grey scale image of the W4 HiI region. The contour starts at the level of $4 \sigma$ with an increment of $1 \sigma$. The blue circles represent the locations of distributed YSOs identified using $24 \mu \mathrm{m}$ flux (for details see text). Plus symbol and dashed circle represent the center and the extent of the cluster IC 1805, respectively (Panwar et al. 2017). Right panel : zoomed-in view (red: IRAC 4.5 $\mu \mathrm{m}$, green : PANSTARR (PS1) y-band and blue : PS1 r-band) of density peaks '3'(right-upper), '4' (right-middle) and ' 5 ' (right-lower). 


$$
\rho(r) \propto \rho_{0}\left\{\left[1+\left(\frac{r}{r_{c}}\right)^{2}\right]^{-0.5}-\left[1+\left(\frac{r_{t}}{r_{c}}\right)^{2}\right]^{-0.5}\right\}^{2.0},
$$

where the parameters $\rho_{0}, r_{c}$ and $r_{t}$ correspond to the central density, core radius and tidal truncation radius, respectively. The core radius $\left(\mathrm{r}_{c}\right)$ provides information about the innermost cluster structure whereas $r_{t}$ and $\gamma$ tell about the outermost region of the cluster. The empirical King profile fitting on the observed RDP is shown with solid black curve in Fig. 4a. In present work, we are only interested in the outer extent of the clusters. The radius where the stellar density profile merges to the background density is defined as the cluster radius $\left(r_{c l}\right)$. Based on the RDP, we find that $r_{c l}$ (where the surface density becomes nearly constant) is about $3^{\prime}$.

A massive star LSI +61 298 (B1 V; Reed 2005) lies within the cluster radius. The radial velocity of the star is $-32.9 \mathrm{~km} / \mathrm{s}$ (Huang \& Gies 2006), consistent with the velocity of the molecular cloud (-55 to $-32 \mathrm{~km} / \mathrm{s}$ ) associated with the W3/W4 complex (Heyer \& Terebey 1998; Carpenter et al. 2000), thus indicating that the cluster is associated with the region.

\subsubsection{Density Peak between CG 7S and IC 1805}

One of the density peaks is near the cometary globule CG 7S $\left(\alpha_{2000}=38^{\circ} .567, \delta_{2000}=+61^{\circ} .421\right)$ which is marked as ' 4 ' in Fig. 3. We also plotted the RDP for this clustering using our YSO sample and fitted with the EFF and King profiles (Fig. 4b). The radial extent of the cluster is $\sim 2$ arcmin. This clustering also contains a massive star LSI +61297 which is designated as a B1 $\mathrm{V}$ type in $S I M B A D$. GAIA has measured a parallax of $0.411 \pm 0.035$ mas for this star, placing it at a similar distance as reported for the HiI region (Gaia Collaboration 2018). The cometary globule CG 7S was extensively studied by Lefloch \& Lazareff (1995) in order to test the RDI model. Using ${ }^{12} \mathrm{CO},{ }^{13} \mathrm{CO}$ and $\mathrm{C}^{18} \mathrm{O}$ observations they found a small dense core hidden by a low density halo. They compared their observations with the numerical simulations of the RDI model and suggested that the globule is in the state of re-expansion, exhibiting the features of the pre-cometary phase. The suggested typical age of the globule was $\sim 1.4 \mathrm{Myr}$ which is less compared to the age $(\sim 2.5 \mathrm{Myr})$ of the cluster IC 1805 .

\subsubsection{Southern density Peak}

Towards the south-east periphery of the HII region, we notice another YSO density peak (marked as ' 5 '; $\alpha_{2000}=39^{\circ} .179, \delta_{2000}=+60^{\circ} .537$ ). The RDP for this clustering yields a cluster radius of $\sim 2.5$ arcmin (see Fig. 4c). This clustering does not contain previously known massive star but there is a massive OB type star $\sim 6$ arcmin away from the cluster center (Reed 2005), probably not the cluster member. The integrated CO (1-0) emission map by Heyer \& Terebey (1998) also shows a strong CO emission towards the south-east side of the HII region which matches with the location of this high density YSOs peak. The $\mathrm{V}_{L S R}$ of this small molecular cloud is $\sim-43 \mathrm{~km} / \mathrm{s}$, consistent with the molecular cloud velocity $(-55$ to $-32 \mathrm{~km} / \mathrm{s}$ ) associated with the $\mathrm{W} 3 / \mathrm{W} 4$ complex (Heyer \& Terebey 1998). The strong CO emission and apparent clumping of YSOs suggests that this cluster is associated with the HII region.

\subsubsection{Evolutionary stages of the newly identified clusters}

To study the evolutionary stages of the new clusters, we used the 2MASS NIR $J$ vs. $(J-H)$ CMDs. The CMDs for the stars in the newly identified clusters are shown in Fig. 5. We note that the YSOs shown in the CMD are biased to the low-extinction region only. Thick black dots are the sources within the radial extents of new clusters. To compare the contribution of field stars in YSO population of the new clusters, we took a field region towards the northern side of the His region. The small grey dots in Fig. 5 represent the sources in the field regions of the same areas as the cluster regions. The star symbols and open circles represent the YSO candidates within the cluster radius and core radius, respectively. In Fig. 5, PMS isochrones of 1 and $10 \mathrm{Myr}$ from Siess et al. (2000) and main sequence (MS) from Girardi et al. (2002), corrected for the adopted distance $(2.0 \mathrm{kpc})$ and average reddening (obtained from the extinction map) are also plotted. The upper and lower dashed lines represent reddening vectors of $A_{V}=10 \mathrm{mag}$ for $4 \mathrm{M}_{\odot}$ and 2 $\mathrm{M}_{\odot}$ PMS star, respectively. The CMDs show that most of the identified YSOs in the newly identified clusters are $\leq 1 \mathrm{Myr}$ old. Due to low statistics of the sources, it is impossible to obtain statistically robust ages of the clusters using $J /(J-H)$ CMD. However, as can be seen from the CMDs, a majority of the YSOs are located beyond 1 Myr PMS isochrone, implying that the ages of the clusters likely to be less than $1 \mathrm{Myr}$. Since most of the YSOs in these clusters are younger compared to the central cluster, i.e., IC 1805, these clusters belong to a different generation of star-formation in the complex. We discuss the origin of such clusters in Sect. 4.2.3.

\subsection{Distributed Young Stellar Population and its Origin}

Figure 3 shows the W4 complex at $22 \mu \mathrm{m}$ along with the YSO candidates identified using $24 \mu \mathrm{m}$ MIPS data. The center of the cluster IC 1805 and its extent are also marked on the image as a plus symbol and the dashed circle, respectively. As can be seen, most of the MIPS identified YSOs show scattered distribution except an enhanced concentration (or group) at the western border of the bubble. Most of these sources are primarily located beyond the cluster radius. We note that in a recent work Sung et al. (2017) suggested that the cluster radius is 

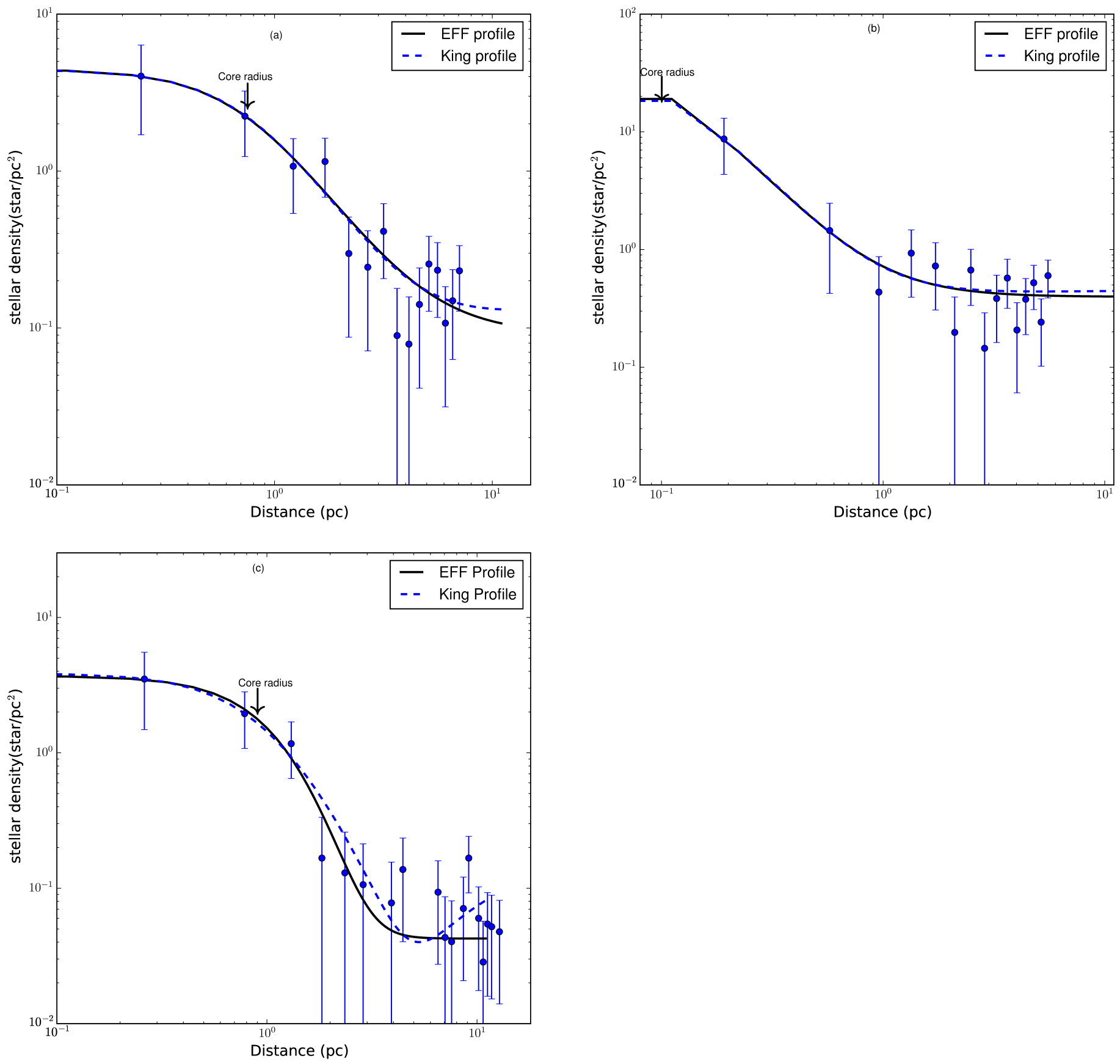

Figure 4. Stellar surface density as a function of radius for the YSOs within the density peaks (black dots) marked as '3' (a), '4' (b), '5' (c) in Fig. 3. Also, the best-fit King profile in solid black and the best-fit EFF profile in dashed blue for the radial profiles of the density peaks are plotted.

likely to be $\sim 15 \operatorname{arcmin}(\sim 10 \mathrm{pc})$. Even if we assume a cluster radius of $10 \mathrm{pc}$, we find $\sim 90 \%$ of the $24 \mu \mathrm{m}$ based class I YSOs located beyond this radius. Among these, around $50 \%$ are likely scattered as they are not spatially coinciding with any clusters found in the surface density map. We note that the group of YSOs found at western border of the bubble are part of a filamentary cloud (for details, see Jose et al. 2016). We also obtained charac- terization of the scattered distribution of the YSOs from the minimum spanning tree (MST) method as explained below.

A scattered and clustered distribution of the sources can be obtained from the MST of the source positions (Schmeja \& Klessen 2006; Gutermuth et al. 2009; Sharma et al. 2016). The MST is defined as the network of lines, or branches, that connect a set of points together such 


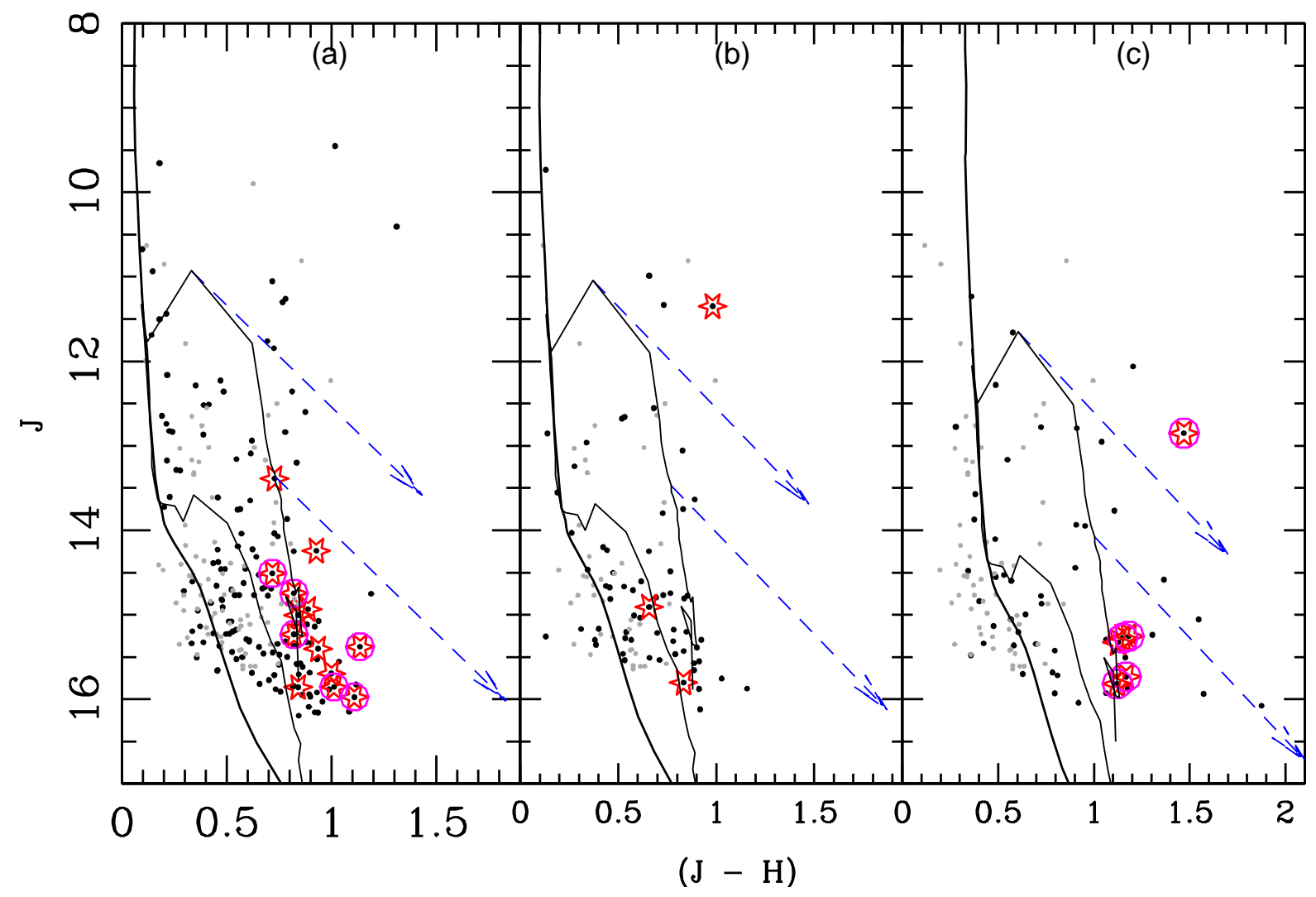

Figure 5. $J$ vs. $(J-H)$ color-magnitude diagrams for the stars within the density peaks (black dots) marked as ' 3 ' (a), '4' (b), '5' (c) in Fig. 3. PMS isochrones from Siess et al. (2000) for 1, 10 Myr and MS from Girardi et al. (2002), corrected for the adopted distance and reddening, are also overplotted. Open circles and red stars represent candidate YSOs within the core radius and cluster radius, respectively. The upper and lower dashed lines are reddening vectors of $\mathrm{A}_{V}=10 \mathrm{mag}$ for $1 \mathrm{Myr}$ PMS stars of $4 \mathrm{M}_{\odot}$ and $2 \mathrm{M}_{\odot}$, respectively.

that the total length of the branches is minimized and there are no closed loops. To obtain the MSTs for the YSOs in the region, we used the approach as suggested by Gutermuth et al. (2009). The histogram of the MST branch length, shown in Fig. 6 (right panel), reveals a peak at short spacing and a long tail towards large spacing. In Fig. 6 (left panel), we plot the cumulative distribution for MST branch lengths. The cumulative distribution function (CDF) shows a three-segment curve; a steep-sloped segment at short spacings, a transition segment that approximates the curved character of the intermediate length spacings, and a shallow-sloped segment at long spacings (cf. Gutermuth et al. 2009). We define a core region where majority of the sources are found in small spacing, i.e., steep segment. We fitted two straight lines to the shallow and steep segments of the $\mathrm{CDF}$ and adopted their intersection as the MST critical branch length (see Fig. 6). The core and the extended regions were isolated by clipping the MST branches longer than the critical length described above. The black dots connected with black lines are the branches smaller than the critical length in the core region. The scattered YSO population (the YSOs outside the cores) clearly show up in the region (see Fig. 7). Since both the above approaches use different methods to identify clusters, some discrepancy on clustering properties is likely. However, all the clusters seen in the surface density map also appear in the MST analysis. Though some new small groups appear in the MST plot, they appear weak in the surface density map. We considered only those groups as cluster that have strong density enhancement in the surface density maps as well as MST plot. We found that the fraction of the distributed YSO population (the YSOs outside the cores) is $\sim 50 \%$ of the total YSOs in the region. If we assume that most of these young sources are part of the W4 complex, it is intriguing how these sources might have formed. Therefore, we evaluate a few processes that can lead to distributed mode of star formation in a star forming complex.

\subsubsection{Hierarchical Fragmentation and Evolution of Molecular Cloud}

In view of the recent numerical simulations of cloud formation and evolution, young star-forming regions are formed by global hierarchical gravitational collapse 

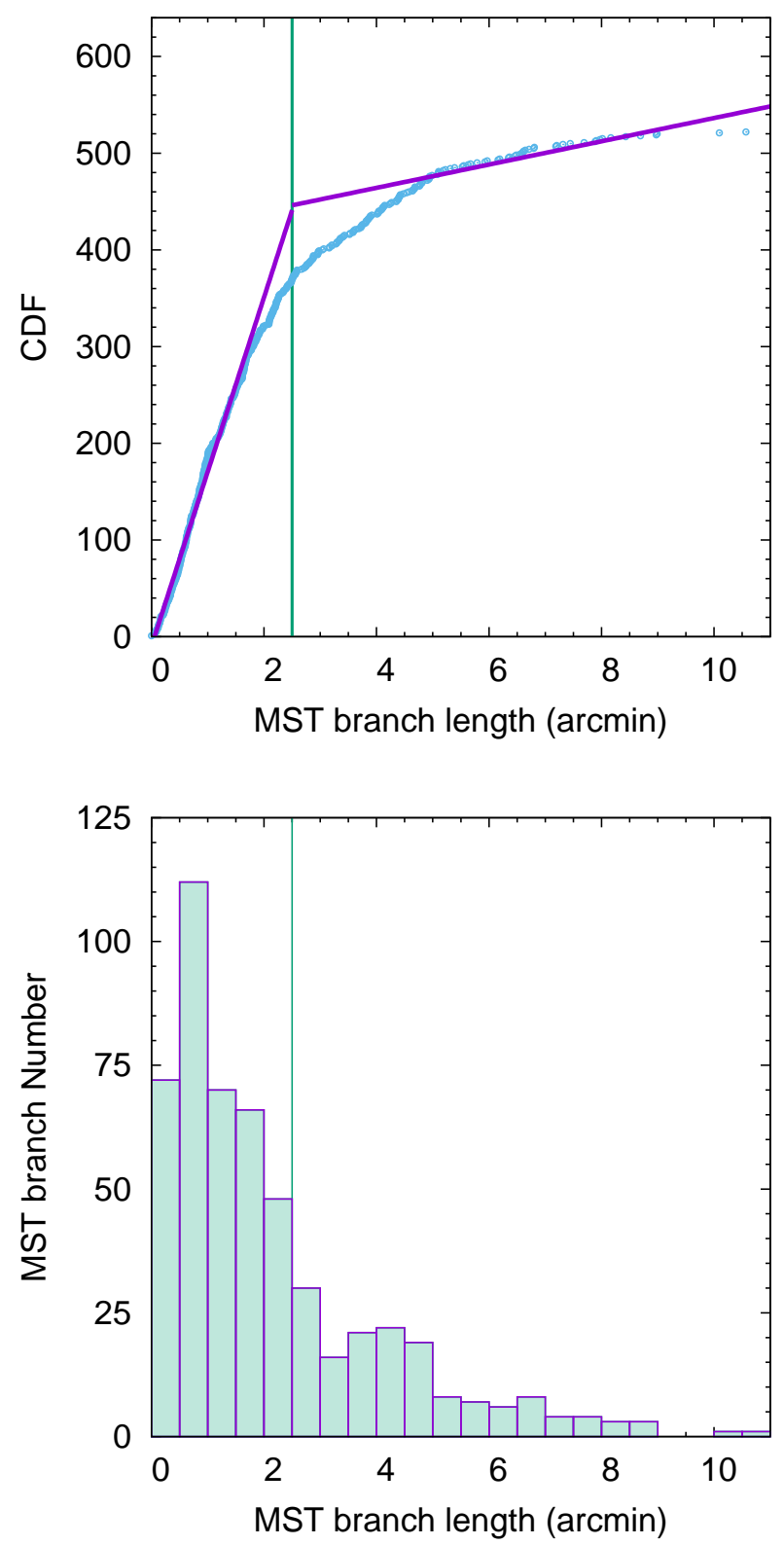

Figure 6. CDF and histogram of MST branch lengths used for critical length analyses of the YSOs. The CDF plot has sorted length values on the $\mathrm{X}$-axis and a rising integer counting index on the $\mathrm{Y}$-axis. The solid line is a two-line fit to the CDF distribution. The vertical line stands for the critical lengths obtained for the core region.

(Vázquez-Semadeni et al. 2009; Zamora-Avilés et al. 2012; Vázquez-Semadeni et al. 2017). In this scenario, as molecular clouds are often turbulent (Klessen et al. 1998; Klessen \& Burkert 2000) the hierarchical structures within the dynamically evolving cloud are created. The free-fall time of any self-gravitating perturbation in the structure is shorter than the overall dynamical time of

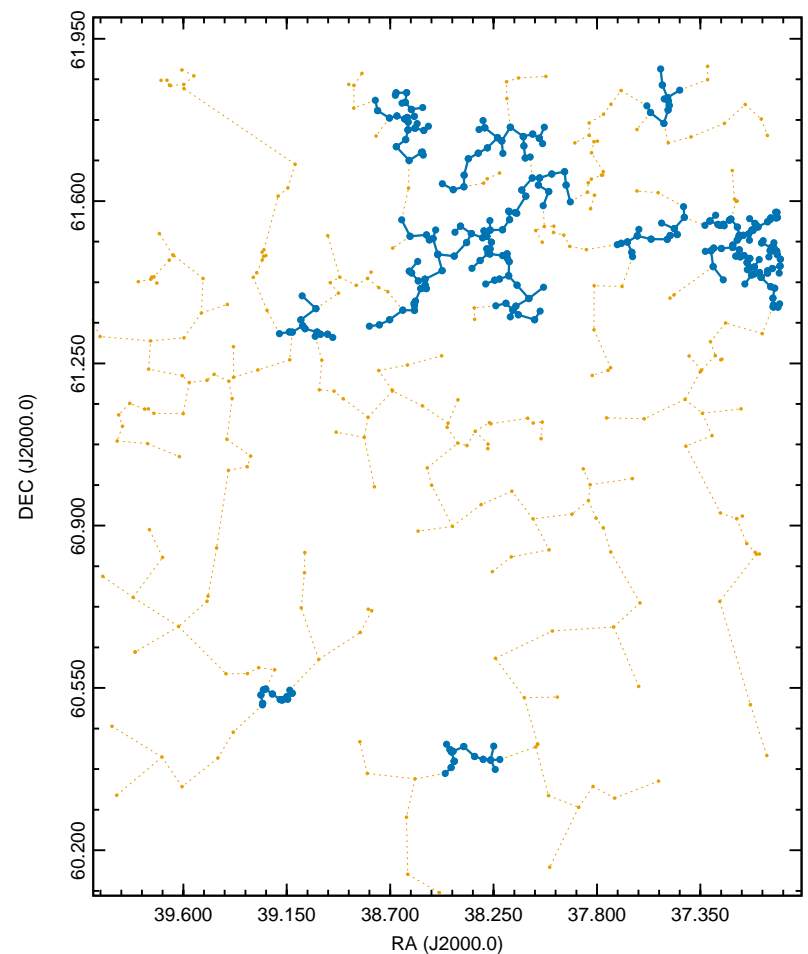

Figure 7. MST for the MIPS identified YSOs in the W4 region. The black dots connected with solid black lines are the branches smaller than the critical length for the cores.

the system. As a result, collapse of molecular cloud is hierarchical, i.e., consisting of small-scale collapses within larger-scale ones. The larger-scale collapse culminate a few Myr later than the small-scale collapse. In other words, star formation occurs in small-scale clump/cores before the onset of star formation in the large-scale clump preferentially located at the centre of the cloud potential. As shown in Vázquez-Semadeni et al. (2017), massive star or cluster is preferentially located at the center of the cloud while the low-mass old stars are located at the outskirts. This process may results in older stars confined to a region where new protostars are forming. Recently, Panwar et al. (2018) also observed a similar age segregation in young cluster Berkeley 59. As shown in the simulations, matter mainly channels to the center of potential through hierarchical network of filaments, thus the older stars formed in the dense structures of the filaments share the in-fall motion of the gas on to the central clump (Vázquez-Semadeni et al. 2017). They have larger velocities and tend to be distributed over larger areas than the younger stars formed in the central clump. If we assume that the aforementioned scenario is responsible for the scattered distribution of the YSOs at the outskirts of the complex, one would expect these sources to be older than the central cluster. Given the fact that 
many YSOs are Class I/II stars of age likely $10^{6} \mathrm{yr}$ (e.g., see discussion in Evans et al. 2009), this process, therefore, should not be a major cause for the origin of such sources.

\subsubsection{Dynamical Ejection of Stars From Cluster Forming Clumps}

Several mechanisms have been proposed for the formation of low-mass stars and brown dwarfs. One of them is the dynamical ejection (Reipurth \& Clarke 2001; Boss 2001; Bate et al. 2002; Goodwin \& Whitworth 2007) which suggests that low-mass objects (e.g., brown dwarfs) form in dense multiple systems, and they are ejected due to dynamical instabilities before they can accrete more mass from their environment. These sources would have a higher velocity dispersion than higher mass objects.

The detections of outflows and disks around brown dwarfs suggest that brown dwarfs and low-mass stars share a similar formation process. If we assume that a fraction of the low-mass stars in a clustered environment followed the ejection scenario proposed by Reipurth \& Clarke (2001) with a resulting average velocity larger than the cluster escape velocity or with a velocity dispersion larger than a few $\mathrm{km} / \mathrm{s}$ as found in simulations, then a large number of low-mass stars are expected to be lost relatively quickly. For example, Kroupa \& Bouvier (2003) suggested that the ejection mechanism could explain a part of the deficit of substellar objects in the Taurus association. Extreme dynamical interactions, namely, close encounters between stars in multiple systems, can produce high-velocity runaway stars. Ejection of massive stars with a high velocity from cluster center has been noted in many cases, e.g., Sterzik \& Durisen (1995) have calculated time-scales for close systems and predicted velocities of $3-4 \mathrm{~km} / \mathrm{s}$ for the ejected stars. Assuming an ejection velocity of $\sim 3 \mathrm{~km} / \mathrm{s}$ perpendicular to the line of sight, a distance of $8.5 \mathrm{pc}$ could be traversed in $3 \mathrm{Myr}$ which is the upper-age limit of the cluster IC 1805. However, in the present work, we find most of the $24 \mu \mathrm{m}$ YSOs are distributed $>17 \mathrm{pc}$ away from the cluster center. Thus it is natural to hypothesize that the low-mass young stars observed at the outskirts of the complex are unlikely the ejected members of the cluster IC 1805. However, if we assume that the low-mass stars are the ejected members of the small-scale cores/clumps that formed during the initial stage of global hierarchical gravitational collapse of the cloud, one would expect these sources to be older compared to stars at the cluster center. However, we acknowledge that without precise knowledge of age and motion of the sources, a conclusive answer can not be given.

\subsubsection{Effect of Stellar Feedbacks: star formation in} small-scale structures such as pillars and globules
Massive stars play a critical role in the evolution of natal molecular cloud by regulating the local ISM through their intense radiation and winds (Lopez et al. 2011; Dale et al. 2013; Walch et al. 2013; Jose et al. 2013; Samal et al. 2014). Recent simulations and observations (e.g., Myers 2009; Schneider et al. 2013; Mallick et al. 2013; Kirk et al. 2014; Samal et al. 2015; Rayner et al. 2017; Dutta et al. 2018) show that cluster formation mainly occurs at the center of cloud potential to which gas flows through a network of threaded filamentary or low-density striation like structures. The erosion of these filaments/accretion flows due to the expanding radiation and winds from the massive stars of the cluster results in elephant-trunk or pillars (Dale et al. 2015). Elephant trunks are long columns of gas and dust that extend radially from the molecular shell driven by the massive stars toward the central star or cluster and have thin bright rim-like structure at their heads. Pillars have typical widths of 0.1 $0.7 \mathrm{pc}$ and size scales of $\sim 0.6-3 \mathrm{pc}$, generally attached to their natal molecular cloud whereas globules are isolated and have a head-tail appearance (Gahm et al. 2006; Schuller et al. 2006; Schneider et al. 2016). Generation of such structures also depends on the fractal dimension of the cloud. For example, using SPH simulations, Walch et al. (2013) have shown that if the fractal dimension of the molecular cloud is low, the border of the HiI region is dominated by extended shell-like structures, which break up into a small number of massive high-density clumps to spawn star clusters. Conversely, if the fractal dimension is high, the border of the HII region is dominated by a large number of pillars and cometary globules, which contain compact dense clumps and tend to spawn single stars or individual multiple systems; the stellar masses are somewhat lower, and the stars are more widely distributed.

The number of such structures in a given complex depends on the hierarchical nature of star-forming cloud. We note that elephant trunk-like structures (ETLSs) are explained based on the models that do not require clumpy material initially (e.g., hydrodynamical instabilities and high curvature in the shell; Chauhan et al. 2011; Tremblin et al. 2012a,b). Since the masses of the clouds that are associated to the ETLSs are often small $(\sim 5-$ $10 \mathrm{M}_{\odot}$ ), they are likely sites of low-mass star formation. Therefore, finding elephant- and pillar-like structures are of great importance for understanding the formation and distribution of low-mass stars in massive star forming complexes.

W4 HiI region powered by several O-type stars of the cluster IC 1805 is also a reservoir for such objects. The stellar feedback has significantly influenced this complex as it contains many BRCs. Out of these BRCs, BRC 5 and BRC 7 are extensively investigated and the smallscale star formation events triggered by the hot mas- 


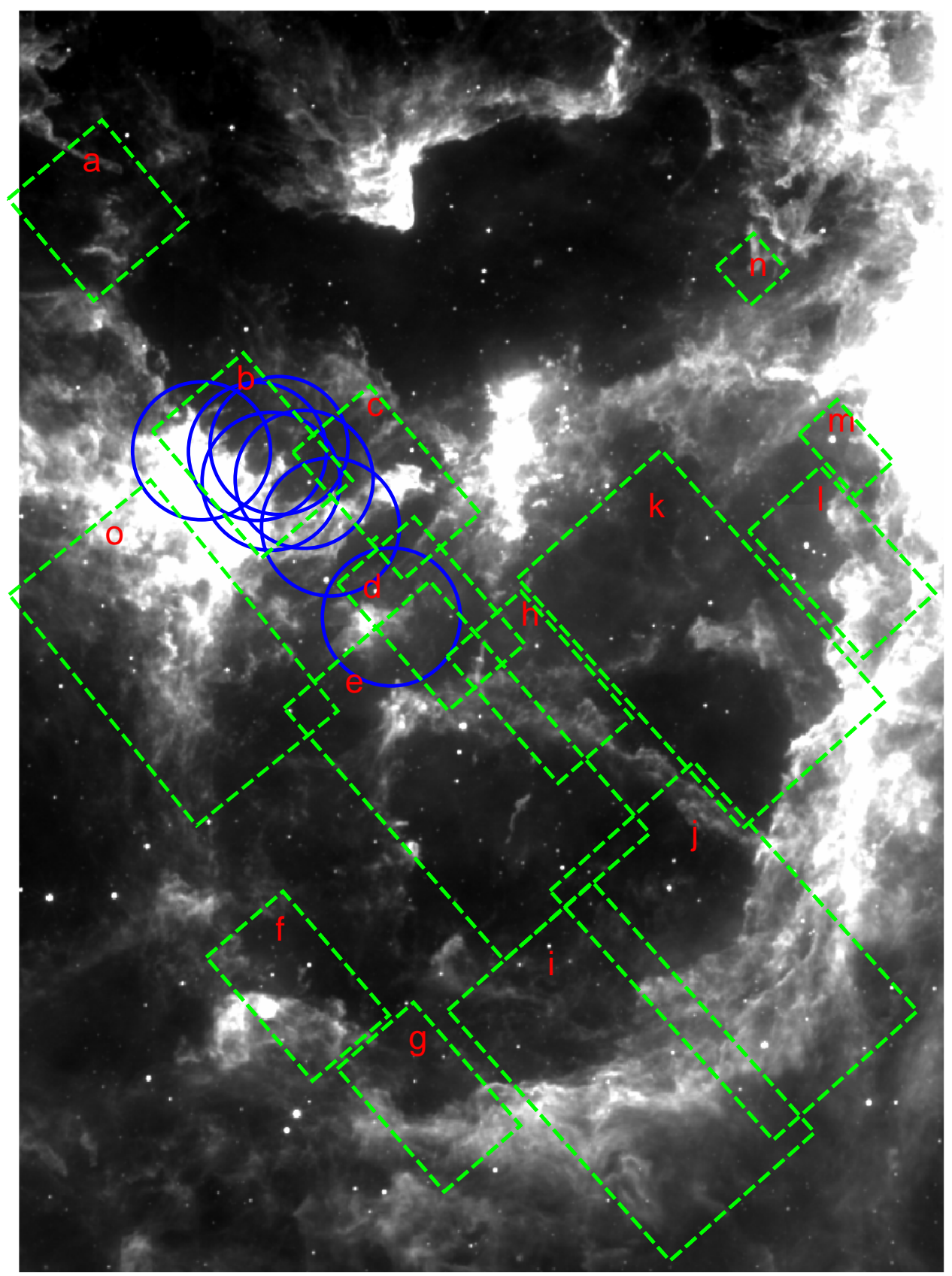

Figure 8. The WISE $12 \mu \mathrm{m}$ image of the HII region. Rectangular regions enclose the ETLSs with stars at their tips. The area covered by Gahm et al. (2007) to identify globulettes are shown with circles.

sive stars are suggested (Ogura et al. 2002; Fukuda et al. 2013; Panwar et al. 2014; Jose et al. 2016). We suggest that the stars associated with these BRCs and the newly identified clusters are a result of the compressing effect of massive stars on the in-falling molecular material to the cluster potential through relatively massive filaments (see simulations of Dale et al. 2012). The MIR/FIR color-composite image of W4 Hil region is shown in Fig. 1. It reveals several thin and elongated ETLSs within the HII region that look morphologically different from usual BRCs. These structures have their tips pointing towards the massive stars, suggesting that their formation and evolution is strongly influenced by the massive members of the cluster IC 1805. Similar structures have been observed in the high-resolution images of many HiI regions taken with the Hubble Space Telescope and the Spitzer Space Telescope (e.g. Hester et al. 1996; Smith et al. 2010; Chauhan et al. 2011).

Due to low-resolution of Herschel images, we used Spitzer images to identify ETLSs. Based on the Spitzer images, we have identified several ETLSs in the complex. Out of these $\sim 24$ ETLSs seems to have stars at 

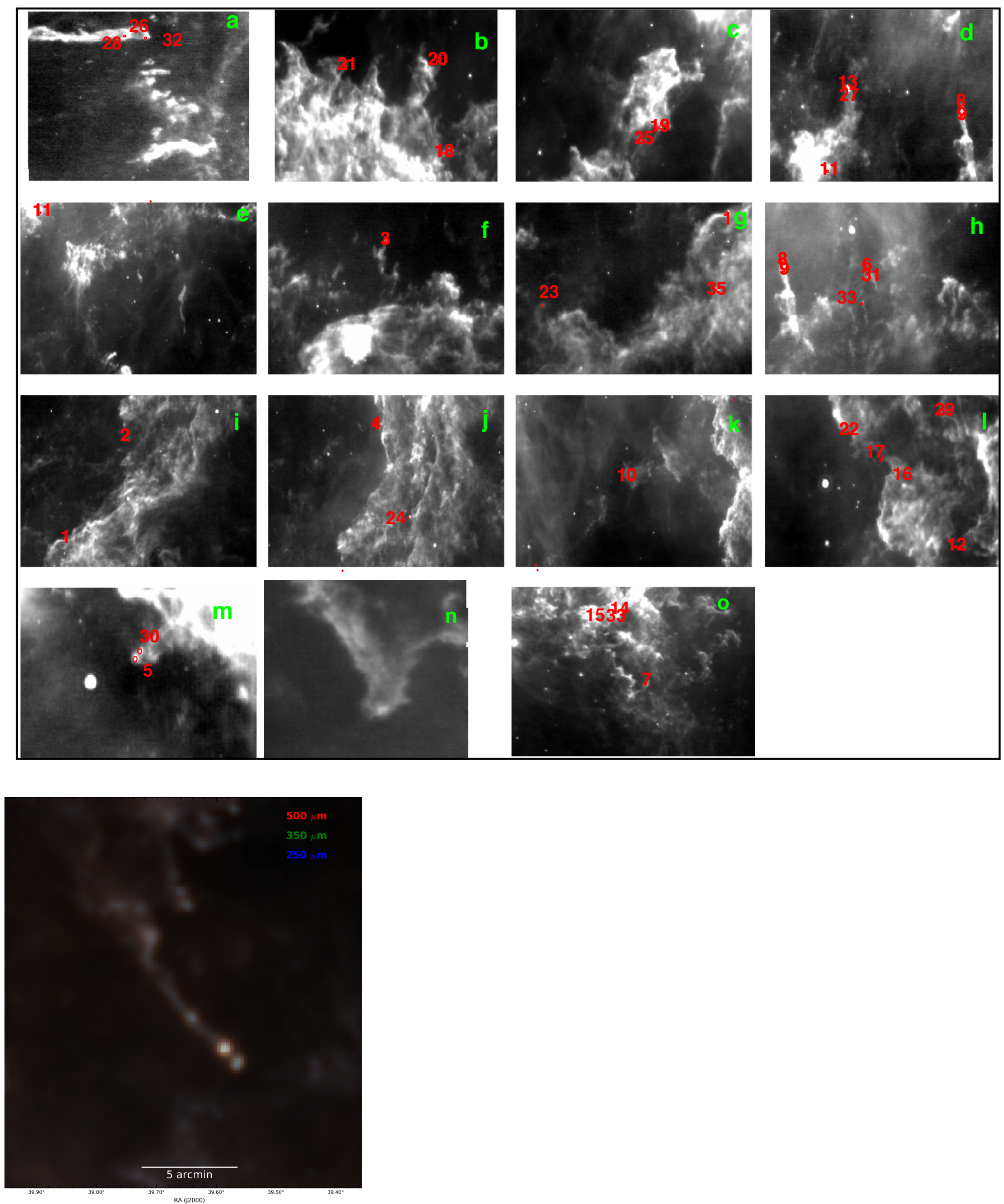

Figure 9. Zoomed-in view of the ETLSs within the rectangular regions of Fig. 8 (upper panel) and an ETLS prominent at longer wavelength with two Class I sources at its tip (lower panel). 


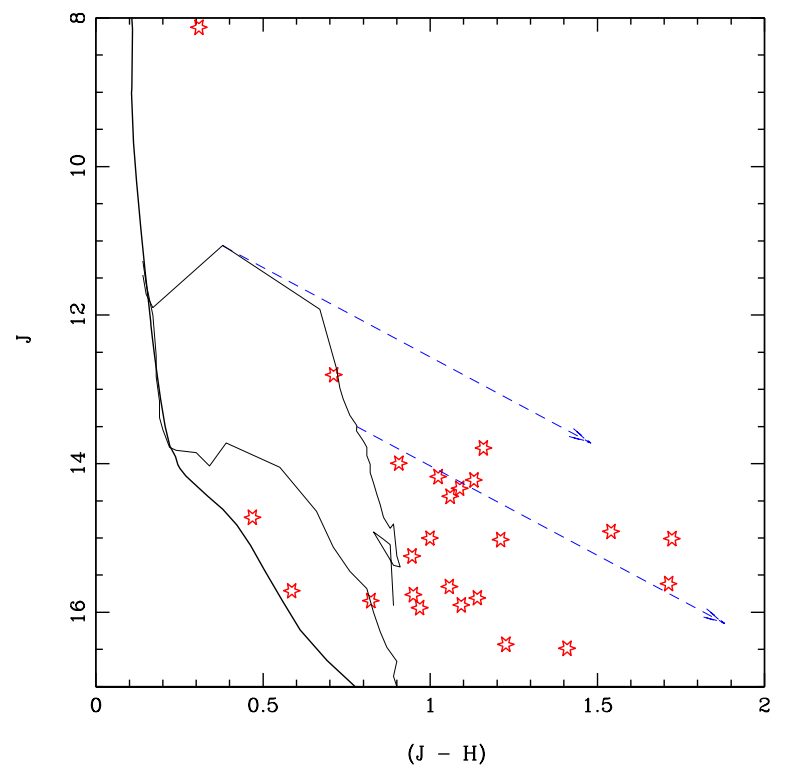

Figure 10. The $J /(J-H)$ CMD for the stars at the tips of ETLSs. PMS isochrones from Siess et al. (2000) for 1, 10 Myr and MS from Girardi et al. (2002), corrected for the adopted distance and reddening, are also overplotted. The upper and lower dashed lines are reddening vectors of $\mathrm{A}_{V}=10 \mathrm{mag}$ for $1 \mathrm{Myr}$ PMS stars of $4 \mathrm{M}_{\odot}$ and 2 $\mathrm{M}_{\odot}$, respectively.

their tips. The locations of the identified ETLSs and the zoomed-in view of the ETLSs alongwith stars at their tips are shown in Fig. 8 \& 9, respectively. A zoomed-in view of one of the ETLSs at longer wavelength is shown in Fig 9b, where one can see two Class I sources at its tip. The positions of the stars at the tips of the ETLSs are catalogued in Table 2 . We note that the ETLSs identified by us are elongated structures with aspect ratios in the range of $\sim 2-7$ and lengths of about $0.4-2.0$ pc. In total we have identified $\sim 38$ ETLSs of lengths greater than 0.4 pc. However, we acknowledge that our identification is biased towards longer ETLSs. We are looking at the ETLS in 2D projection, thus their sizes and total numbers are likely an underestimation. We also note that the cold gas content of many small-scales ETLSs might have been completely cleared by the UV photons of the massive stars. Therefore, some of the YSOs seen today without associated pillars could be part of such a process.

Chauhan et al. (2011) found YSOs at the tips of many ETLSs in W5 E HII region. Based on the optical CMDs and SED fitting of these stars, they found mass $<2 \mathrm{M}_{\odot}$ for most of the stars. In order to determine the masses of the YSOs located at the tips of the ETLSs identified in the present work, we used $J$ vs. $(J-H)$ diagram. This

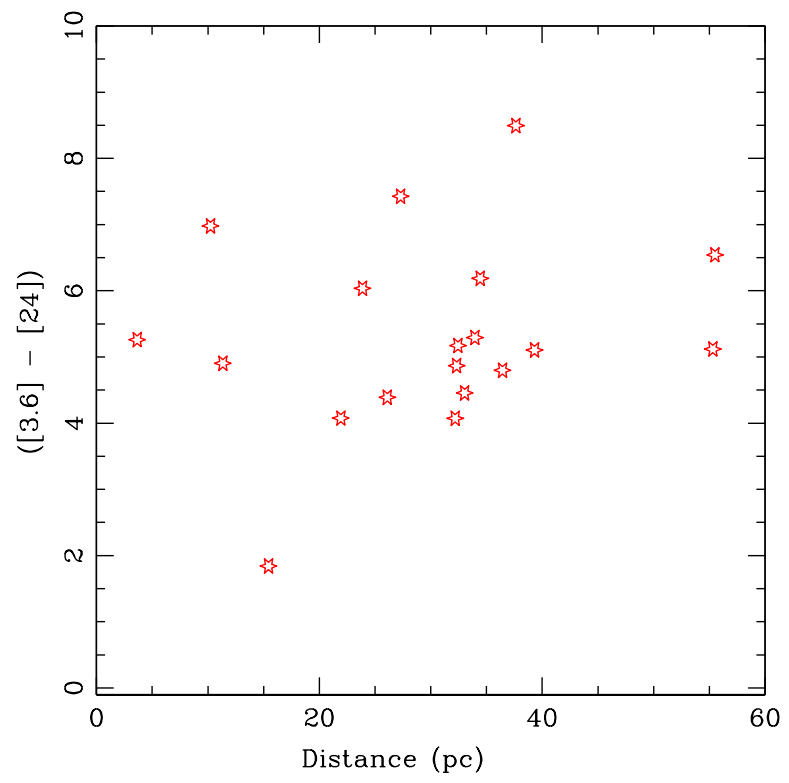

Figure 11. The ([3.6] - [24]) colours of the ETLSs stars as a function of radial distance from the center of the cluster IC 1805. Most of these sources have [3.6]-[24] color greater than 4.5, thus are likely protostars (e.g., see Gutermuth et al. 2009).

is because among near-infrared bands, the effect of the excess emission on the mass estimation due to circumstellar disk in the J-band is minimal (e.g., Meyer et al. 1997; Luhman et al. 2003). Fig. 10 shows $J$ vs. $(J-H)$ CMD of the stars at the tips of the ETLSs. For comparison, we have also over-plotted the PMS isochrones for 1 Myr and 10 Myr from Siess et al. (2000) and MS from Girardi et al. (2002), corrected for the adopted distance and average reddening. The location of the stars in the CMD clearly indicates that most of them are very young $(<1$ Myr old) low-mass objects. Fig. 11 shows the ([3.6] - [24]) color vs. distance (from cluster center) of the stars associated with the ETLSs. As can be seen, stars at the tips of ETLSs identified in the present work are lying at a distance range of 5 to 90 arcmin from the cluster center, with majority beyond $30 \operatorname{arcmin}(\sim 17 \mathrm{pc})$. Their distribution suggests that most of these young stars are lying at the outskirts of the complex. If it is to be believed that these stars are formed due to compression and squeezing of pre-existing material like star formation in BRCs, it can be argued that small-scale star formation is currently occurring in the outer extent of the cloud via triggering.

As mentioned earlier, our identification is biased towards longer ETLSs that are visible as thin long structures pointing towards massive stars from the base of the parental cloud or bubble wall in the $24 \mu \mathrm{m}$ maps above 
background emission. Therefore, our catalog lacks smallscale structures such as globulettes which are very small and somewhat round dark clouds (Gahm et al. 2007) and isolated knots that are detached from the parental clouds. It is worth noting that using narrow-band $\mathrm{H} \alpha$ observations taken with 2.6m Nordic Optical Telescope, La Palma, Carlqvist et al. (2003) \& Gahm et al. (2007) have found $\sim 18$ globulettes within the circular regions shown in Fig. 8. They investigated the position, dimension, orientation of the globulettes, and found that most objects were smaller than $9 k A U(\sim 0.04 \mathrm{pc})$ and nearly round in shape. Many of them were associated with dense cores which might collapse to form planetary-mass objects.

As we find only one star or at most a few stars at the tip of each ETLS, the scale of star formation in each cloud associated with ETLSs is very small. However, the total product can be considerable because a large number of such structures can be formed in Hil region environment (e.g., see simulation results of Walch et al. 2013). We note that recent studies based on the Herschel observations suggest that filaments and filament structures of lengths similar to that of ETLSs are ubiquitous in star forming clouds (e.g. Arzoumanian et al. 2011; Malinen et al. 2012; Palmeirim et al. 2013; André et al. 2014). Numerical works show that filaments are prone to fragmentation due to their geometry where small-scale density perturbations have time to collapse locally before global collapse occurs (Pon et al. 2011). Therefore, it is difficult to conclude whether these aggregates are really due to triggering, or they are simply the sources of the filaments uncovered by the expanding Hil region. However, the circumstantial evidence favors the former. For example, in general it has been found that young protostars or cores are distributed along the long axis of the filament more or less like beads strung on a wire (Tafalla \& Hacar 2015; Anathpindika \& Freundlich 2015; Samal et al. 2015). In contrast, we find a single or at best a few protostars only at the apex of the ETLSs facing the massive cluster; indicating their formation is very likely influenced by the comprehensive effect of expanding HiI regions on the cold materiel of the ETLSs. It is difficult to conclude whether this effect is weak (i.e., temporarily increasing the star formation rate by inducing stars to form earlier) or strong (i.e., increasing the star formation efficiency by causing the birth of stars that would not otherwise form). High resolution sensitive observations of the W4 complex at infrared and millimeter bands, along with proper-motion and age measurements of the stars would reveal the prevalence of such structures and scale of star-formation within them. Nonetheless, taking the results of the present work at face value it can be argued that star formation in small-scale structures such as pillars or elephant trunks or globulettes is likely an important ingredient for the formation of low-mass
Table 2. Location and nature of the sources at the tips of ETLSs in the W4 complex.

\begin{tabular}{cccc}
\hline S. No. & RA $(\mathrm{J} 2000)$ & Dec $(\mathrm{J} 2000)$ & Evolutionary stage \\
\hline 1 & 38.379875 & 60.423753 & Class I \\
2 & 37.973150 & 60.530311 & Class II \\
3 & 39.010692 & 60.611611 & Class II \\
4 & 37.612908 & 60.733211 & Class II \\
5 & 37.250650 & 61.430108 & Class I \\
6 & 38.274375 & 61.076200 & Class II \\
7 & 39.410421 & 61.086119 & Class II \\
8 & 38.448054 & 61.120731 & Class I \\
9 & 38.451850 & 61.112100 & Class II \\
10 & 37.758258 & 61.132481 & Class II \\
11 & 38.795842 & 61.133550 & Class II \\
12 & 37.173542 & 61.151639 & Class II \\
13 & 38.691429 & 61.192431 & Class II \\
14 & 39.380979 & 61.220169 & Class II \\
15 & 39.466100 & 61.226253 & Class II \\
16 & 37.256721 & 61.258931 & Class II \\
17 & 37.261350 & 61.257700 & Class II \\
18 & 39.138429 & 61.317725 & Class I \\
19 & 38.747588 & 61.333089 & Class II \\
20 & 39.083142 & 61.395622 & Class II \\
21 & 39.295542 & 61.435703 & Class I \\
22 & 37.306521 & 61.296750 & Class I \\
23 & 38.832171 & 60.434014 & Class I \\
24 & 37.618654 & 60.553192 & Class I \\
25 & 38.790225 & 61.329911 & Class I \\
26 & 39.671125 & 61.860408 & Class I \\
27 & 38.690279 & 61.190503 & Class I \\
28 & 39.696529 & 61.860408 & Class I \\
29 & 37.088583 & 61.266072 & Class I \\
30 & 37.242608 & 61.432064 & Class I \\
31 & 38.275258 & 61.066281 & Class II \\
32 & 39.655446 & 61.849247 & Class II \\
33 & 37.565021 & 61.696329 & Class I \\
34 & 36.338496 & 60.966117 & Class I \\
35 & 38.460296 & 60.365197 & Class I \\
36 & 39.927787 & 62.012761 & Class I \\
\hline & & &
\end{tabular}

stars at the outskirts of star-forming complexes. Therefore, it could be one of the possible viable mechanisms for forming isolated low-mass stars in molecular clouds.

\section{CONCLUSIONS}

In order to study the clustered and distributed mode of star formation in the W4 HII region, we identified young stellar aggregates using homogeneous datasets from 2MASS, Spitzer and WISE observations. We searched for signatures of star formation in the surroundings and investigated a probable scenario of triggered star formation at the border of the nebula. The spatial density distribution of the YSOs revealed three new clusterings/aggregates in the region, near BRC 7, CG 7S and 
south-east periphery of the HII region. Most of the identified YSOs in these three clusterings are low-mass stars with ages $\leq 1$ Myr. We also noticed many ETLSs in the HII region and most of them have young stars at their tips. We suggest that these may be the product of the interaction of the I/S-fronts with the dynamically evolving clumpy molecular cloud and that distributed low-mass young stars found at the outskirts of the large-complexes may be outcomes of feedback effects of massive stars.

We thank the anonymous referee for the thorough reading of the manuscript and providing useful comments to improve it. NP acknowledges the financial support from the Department of Science \& Technology, INDIA, through INSPIRE faculty award IFA-PH-36. This publication makes use of data from the Two Micron All Sky Survey (a joint project of the University of Massachusetts and the Infrared Processing and Analysis Center/ California Institute of Technology, funded by the National Aeronautics and Space Administration and the National Science Foundation), archival data obtained with the Spitzer Space Telescope and Wide Infrared Survey Explorer (operated by the Jet Propulsion Laboratory, California Institute of Technology, under contract with the NASA.

\section{REFERENCES}

Allen, L. E., Calvet, N., D'Alessio, P., et al. 2004, ApJS, 154, 363

Anathpindika, S., \& Freundlich, J. 2015, PASA, 32, e007

André, P., Di Francesco, J., Ward-Thompson, D., et al. 2014, Protostars and Planets VI, 27

Arzoumanian, D., André, P., Didelon, P., et al. 2011, A\&A, 529, L6

Bate, M. R., Bonnell, I. A., \& Bromm, V. 2002, MNRAS, 332, L65

Bernard, A., Neichel, B., Samal, M. R., et al. 2016, A\&A, 592, A77

Bisbas, T. G., Wünsch, R., Whitworth, A. P., Hubber, D. A., \& Walch, S. 2011, ApJ, 736, 142

Boss, A. P. 2001, ApJL, 551, L167

Cambrésy, L., Beichman, C. A., Jarrett, T. H., \& Cutri, R. M. 2002, AJ, 123, 2559

Carlqvist, P., Gahm, G. F., \& Kristen, H. 2003, A\&A, 403, 399

Carpenter, J. M., Heyer, M. H., \& Snell, R. L. 2000, ApJS, 130, 381

Casertano, S., \& Hut, P. 1985, ApJ, 298, 80

Chauhan, N., Ogura, K., Pandey, A. K., Samal, M. R., \& Bhatt, B. C. 2011, PASJ, 63, 795

Cutri, R. M., Skrutskie, M. F., van Dyk, S., et al. 2003, 2MASS All Sky Catalog of point sources.

Cutri, R. M., Wright, E. L., Conrow, T., et al. 2014, VizieR Online Data Catalog, 2328

Dale, J. E., Ercolano, B., \& Bonnell, I. A. 2012, MNRAS, 424, 377

-. 2013, MNRAS, 430, 234

Dale, J. E., Haworth, T. J., \& Bressert, E. 2015, MNRAS, 450, 1199

Deharveng, L., Zavagno, A., Anderson, L. D., et al. 2012, A\&A, 546, A74
Deharveng, L., Zavagno, A., Samal, M. R., et al. 2015, A\&A, 582, A1

Dutta, S., Mondal, S., Samal, M. R., \& Jose, J. 2018, ApJ, 864, 154

Elmegreen, B. G., \& Efremov, Y. N. 1997, ApJ, 480, 235

Elmegreen, D. M., Elmegreen, B. G., Adamo, A., et al. 2014, ApJL, 787, L15

Elson, R. A. W., Fall, S. M., \& Freeman, K. C. 1987, ApJ, 323, 54

Evans, II, N. J., Allen, L. E., Blake, G. A., et al. 2003, PASP, 115,965

Evans, II, N. J., Dunham, M. M., Jørgensen, J. K., et al. 2009, ApJS, 181, 321

Flaherty, K. M., Pipher, J. L., Megeath, S. T., et al. 2007, ApJ, 663,1069

Fukuda, N., Miao, J., Sugitani, K., et al. 2013, ApJ, 773, 132

Gahm, G. F., Carlqvist, P., Johansson, L. E. B., \& Nikolić, S. 2006, A\&A, 454, 201

Gahm, G. F., Grenman, T., Fredriksson, S., \& Kristen, H. 2007, AJ, 133, 1795

Gaia Collaboration. 2018, VizieR Online Data Catalog, 1345

Girardi, L., Bertelli, G., Bressan, A., et al. 2002, A\&A, 391, 195

Goodwin, S. P., \& Whitworth, A. 2007, A\&A, 466, 943

Gutermuth, R. A., Megeath, S. T., Myers, P. C., et al. 2009, ApJS, 184, 18

Gutermuth, R. A., Megeath, S. T., Pipher, J. L., et al. 2005, ApJ, 632,397

Gutermuth, R. A., Myers, P. C., Megeath, S. T., et al. 2008, ApJ, 674,336

Hester, J. J., Scowen, P. A., Sankrit, R., et al. 1996, AJ, 111, 2349

Heyer, M. H., \& Terebey, S. 1998, ApJ, 502, 265

Huang, W., \& Gies, D. R. 2006, ApJ, 648, 580

Jose, J., Kim, J. S., Herczeg, G. J., et al. 2016, ApJ, 822, 49

Jose, J., Pandey, A. K., Samal, M. R., et al. 2013, MNRAS, 432, 3445

King, I. 1962, AJ, 67, 471

Kirk, H., Offner, S. S. R., \& Redmond, K. J. 2014, MNRAS, 439, 1765

Klessen, R. S., \& Burkert, A. 2000, ApJS, 128, 287

Klessen, R. S., Burkert, A., \& Bate, M. R. 1998, ApJL, 501, L205

Koenig, X. P., Allen, L. E., Gutermuth, R. A., et al. 2008, ApJ, 688,1142

Koenig, X. P., Leisawitz, D. T., Benford, D. J., et al. 2012, ApJ, 744,130

Kroupa, P., \& Bouvier, J. 2003, MNRAS, 346, 369

Lefloch, B., \& Lazareff, B. 1995, A\&A, 301, 522

Lopez, L. A., Krumholz, M. R., Bolatto, A. D., Prochaska, J. X., \& Ramirez-Ruiz, E. 2011, ApJ, 731, 91

Luhman, K. L., Stauffer, J. R., Muench, A. A., et al. 2003, ApJ, 593, 1093

Malinen, J., Juvela, M., Rawlings, M. G., et al. 2012, A\&A, 544, A50

Mallick, K. K., Kumar, M. S. N., Ojha, D. K., et al. 2013, ApJ, 779,113

Massey, P., Johnson, K. E., \& Degioia-Eastwood, K. 1995, ApJ, 454, 151

Megeath, S. T., Allgaier, E., Young, E., et al. 2009, AJ, 137, 4072

Megeath, S. T., Allen, L. E., Gutermuth, R. A., et al. 2004, ApJS, 154, 367

Megeath, S. T., Gutermuth, R., Muzerolle, J., et al. 2012, AJ, 144, 192

Meyer, M. R., Calvet, N., \& Hillenbrand, L. A. 1997, AJ, 114, 288

Miao, J., White, G. J., Thompson, M. A., \& Nelson, R. P. 2009, ApJ, 692, 382

Morgan, L. K., Thompson, M. A., Urquhart, J. S., White, G. J., \& Miao, J. 2004, A\&A, 426, 535

Myers, P. C. 2009, ApJ, 706, 1341

Ogura, K., Sugitani, K., \& Pickles, A. 2002, AJ, 123, 2597 
Palmeirim, P., André, P., Kirk, J., et al. 2013, A\&A, 550, A38

Pandey, A. K., Samal, M. R., Chauhan, N., et al. 2013a, New Astronomy, 19, 1

Pandey, A. K., Eswaraiah, C., Sharma, S., et al. 2013b, ApJ, 764, 172

Panwar, N., Chen, W. P., Pandey, A. K., et al. 2014, MNRAS, 443, 1614

Panwar, N., Pandey, A. K., Samal, M. R., et al. 2018, AJ, 155, 44

Panwar, N., Samal, M. R., Pandey, A. K., et al. 2017, MNRAS, 468, 2684

Pon, A., Johnstone, D., \& Heitsch, F. 2011, ApJ, 740, 88

Rayner, T. S. M., Griffin, M. J., Schneider, N., et al. 2017, A\&A, 607, A22

Reed, C. 2005, VizieR Online Data Catalog, 5125

Reipurth, B., \& Clarke, C. 2001, AJ, 122, 432

Robitaille, T. P., Meade, M. R., Babler, B. L., et al. 2008, AJ, 136, 2413

Samal, M. R., Deharveng, L., Zavagno, A., et al. 2018, A\&A, 617, A67

Samal, M. R., Zavagno, A., Deharveng, L., et al. 2014, A\&A, 566, A122

Samal, M. R., Ojha, D. K., Jose, J., et al. 2015, A\&A, 581, A5

Schmeja, S., \& Klessen, R. S. 2006, A\&A, 449, 151

Schneider, N., André, P., Könyves, V., et al. 2013, ApJ, 766, L17

Schneider, N., Bontemps, S., Motte, F., et al. 2016, A\&A, 591, A40

Schuller, F., Leurini, S., Hieret, C., et al. 2006, A\&A, 454, L87

Sharma, S., Pandey, A. K., Ojha, D. K., et al. 2017, MNRAS, 467,2943
Sharma, S., Pandey, A. K., Borissova, J., et al. 2016, AJ, 151, 126

Siess, L., Dufour, E., \& Forestini, M. 2000, A\&A, 358, 593

Smith, N., Povich, M. S., Whitney, B. A., et al. 2010, MNRAS, 406, 952

Snider, K. D., Hester, J. J., Desch, S. J., Healy, K. R., \& Bally, J. 2009, ApJ, 700, 506

Sterzik, M. F., \& Durisen, R. H. 1995, A\&A, 304, L9

Sugitani, K., Fukui, Y., \& Ogura, K. 1991, ApJS, 77, 59

Sung, H., Bessell, M. S., Chun, M.-Y., et al. 2017, ApJS, 230, 3

Tafalla, M., \& Hacar, A. 2015, A\&A, 574, A104

Tremblin, P., Audit, E., Minier, V., Schmidt, W., \& Schneider, N. 2012a, A\&A, 546, A33

Tremblin, P., Audit, E., Minier, V., \& Schneider, N. 2012b, A\&A, 538, A31

Vázquez-Semadeni, E., Gómez, G. C., Jappsen, A.-K., Ballesteros-Paredes, J., \& Klessen, R. S. 2009, ApJ, 707, 1023

Vázquez-Semadeni, E., González-Samaniego, A., \& Colín, P. 2017, MNRAS, 467, 1313

Walch, S., Whitworth, A. P., Bisbas, T. G., Wünsch, R., \& Hubber, D. A. 2013, MNRAS, 435, 917

Yadav, R. K., Pandey, A. K., Sharma, S., et al. 2016, MNRAS, 461, 2502

Zamora-Avilés, M., Vázquez-Semadeni, E., \& Colín, P. 2012, ApJ, 751,77 